\title{
A rigorous analytical method for doubly periodic cylindrical inclusions under longitudinal shear and its application
}

\author{
C.P. Jiang ${ }^{\text {a,b }}$, Y.L. Xu ${ }^{\text {b }}$, Y.K. Cheung ${ }^{c, *}$, S.H. Lo ${ }^{c}$ \\ ${ }^{a}$ LNM, Institute of Mechanics, Chinese Academy of Sciences, Beijing 100080, China \\ ${ }^{\mathrm{b}}$ Solid Mechanics Research Center, Beijing University of Aeronautics and Astronautics, Beijing 100083, China \\ ${ }^{\mathrm{c}}$ Department of Civil Engineering, The University of Hong Kong, Pokfulam Road, Hong Kong, China
}

Received 28 August 2002; received in revised form 1 December 2002

\begin{abstract}
An infinite elastic solid containing a doubly periodic parallelogrammic array of cylindrical inclusions under longitudinal shear is studied. A rigorous and effective analytical method for exact solution is developed by using Eshelby's equivalent inclusion concept integrated with the new results from the doubly quasi-periodic Riemann boundary value problems. Numerical results show the dependence of the stress concentrations in such heterogeneous materials on the periodic microstructure parameters. The overall longitudinal shear modulus of composites with periodic distributed fibers is also studied. Several problems of practical importance, such as those of doubly periodic holes or rigid inclusions, singly periodic inclusions and single inclusion, are solved or resolved as special cases. The present method can provide benchmark results for other numerical and approximate methods.
\end{abstract}

(C) 2003 Elsevier Ltd. All rights reserved.

Keywords: Double period; Riemann boundary value problem; Heterogeneous materials; Fiber-reinforced composites; Effective modulus; Stress concentration

\section{Introduction}

Macroscopic behaviors of heterogeneous materials to a great extent depend on their microstructure pattern. Engineers continue to develop better devices and structures. One of the key ways to achieve optimization is by tailoring the materials making up a device to best serve the intended

\footnotetext{
${ }^{*}$ Corresponding author. Tel.: +852-2859-2668; fax: +8522559-5337.

E-mail address: hreccyk@hkucc.hku.hk (Y.K. Cheung).
}

application. This trend began with the introduction of composite materials and may well continue with the creation of completely novel materials by direct engineering of their microstructure (Tadmor et al., 2000), which has resulted in a strong drive towards the study of micromechanics for heterogeneous media. Many natural, and especially manmade materials, possess periodic or approximately periodic microstructures. Micromechanics for solids with periodic microstructures has attracted the attention of many scholars, and the references (Tsai, 1966; Chen, 1970; Furuhashi et al., 1981; Nemat-Nasser and Taya, 1981, 1985; Nunan and 
Keller, 1984; Accorsi and Nemat-Nasser, 1986; Walker et al., 1991; Helsing, 1995; RodríguezRamos et al., 2001; Bravo-Castillero et al., 2001) provide examples of contributions to solids with periodic inclusions. The theory and method of micromechanics for solids with periodic microstructures are developed in Nemat-Nasser and Hori's book (1999), where the general solution is obtained by using the Fourier series expansion technique. For the special case of an infinitely extended homogeneous linearly elastic solid containing an ellipsoidal inclusion, the important results were given by Eshelby (1957), and the corresponding exact eigenstrain is obtained. However, in general, it is a difficult task to determine the exact Fourier coefficient of the corresponding eigenstrain for solids with periodic inclusions (see Nemat-Nasser and Taya (1981, 1985) and Nunan and Keller (1984) for discussion of alternative methods of solution). A good approximation may be obtained if the eigenstrain is replaced by its average value and the result is entered into the consistency condition (NematNasser and Hori, 1999).

The complex variable method as well as the theory of boundary value problems for analytical functions (Muskhelishvili, 1956, 1958) are powerful tools for 2-dimensional heterogeneous materials. The doubly periodic Riemannn boundary value problem was studied first by Chibrikova (1956), but her results are not valid for the general case, and later she made a correction (Chibrikova, 1970). Lu (1993) presented a method which is valid for the general case, and Li (1999) studied applications of doubly quasi-periodic boundary value problems in elasticity theory. However, to our knowledge, effective numerical methods for heterogeneous materials based the elegant theory of doubly quasi-periodic Riemann boundary problems have not been reported yet.

This paper constitutes a continuing study in the quest for rigorous and efficient analytical methods for heterogeneous materials with periodic microstructures and in understanding the behaviors of such materials. Using the equivalent inclusion concept (Eshelby, 1957; Nemat-Nasser and Hori, 1999) and integrated with the new results from doubly quasi-periodic Riemann boundary value problems (Lu, 1993), a rigorous and efficient analytical method is developed for the problem of doubly periodic cylindrical inclusions under longitudinal shear. Illustrative examples are given, and the influence of fiber array on local stress concentrations and overall properties of composite materials is examined.

\section{Statement of the problem}

Fig. 1 is a schematic diagram of doubly periodic cylindrical inclusions (cross-section) in an infinite matrix subjected to a uniform longitudinal shear stress at infinity, $\tau_{x z}^{\infty}=q_{1}, \tau_{y z}^{\infty}=q_{2}$ (which is not depicted in the figure). The moduli of the matrix and inclusions are $G$ and $G^{\prime}$, respectively, and their interfaces are perfectly bonded. In the complex plane $z=x+\mathrm{i} y, 2 \omega_{1}$ and $2 \omega_{2}$ denote two fundamental periods with $\operatorname{Im} \omega_{2} / \omega_{1}>0$, where Im denotes the imaginary part. $P_{00}$ is the fundamental parallelogram (one of the largest parallelograms without congruent points) or fundamental cell

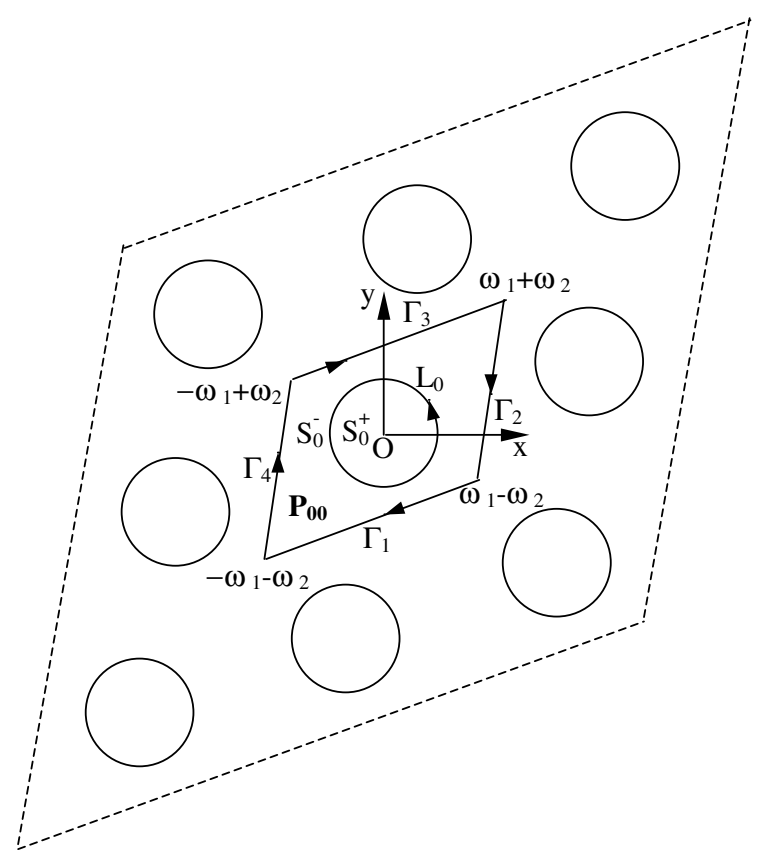

Fig. 1. A schematic diagram of doubly periodic array cylinder inclusions in an infinite matrix. 
with the boundary $\Gamma=\Gamma_{1}+\Gamma_{2}+\Gamma_{3}+\Gamma_{4}$ oriented in the closewise direction and the vertices $\omega_{1}+\omega_{2},-\omega_{1}+\omega_{2},-\omega_{1}-\omega_{2}$ and $\omega_{1}-\omega_{2}$. Without loss of generality, assume the fundamental cell and the circular inclusion within it share the common center (the origin), as otherwise, a suitable coordinate translation may be introduced. Let $S_{0}^{+}$denote the region occupied by the inclusion bounded by the contour $L_{0}$ oriented counter-clockwise, $S_{0}^{-}=P_{00}-S_{0}^{+}$and $S^{ \pm}$represent the union of $S_{0}^{ \pm}$and its periodically congruent regions, and $L$ represents $L_{0}$ and its periodically congruent contours.

\section{Equivalent homogeneous solid and eigenstrain}

To formulate the problem, the concept of the equivalent homogeneous solid and eigenstrain are introduced (refer to Nemat-Nasser and Hori, 1999).

Consider an infinite homogeneous linearly elastic solid with the longitudinal shear modulus $G$ (the same as the matrix) subjected to a uniform longitudinal shear stress $\tau_{x z}^{\infty}=q_{1}, \tau_{y z}^{\infty}=q_{2}$. In the entire plane, the stress field $\left(\tau_{x z}, \tau_{y z}\right)$ and hence the corresponding strain field $\left(\gamma_{x z}, \gamma_{y z}\right)$ would be uniform; these fields would be given by $\tau_{x z}^{0}=q_{1}$, $\tau_{y z}^{0}=q_{2} ; \gamma_{x z}^{0}=q_{1} / G, \gamma_{y z}^{0}=q_{2} / G$, respectively. For the actual heterogeneous solid under consideration, the presence of the doubly periodic region $S^{+}$with a different elasticity, i.e., the existence of a material mismatch, disturbs the uniform stress and strain fields. Denote the disturbance stress and strain fields by $\tau_{x z, \text { in }}^{\prime}, \tau_{y z, \text { in }}^{\prime}, \gamma_{x z, \text { in }}^{\prime}, \gamma_{y z \text {,in }}^{\prime}$ in $S^{+} ; \tau_{x z, \text { out }}^{\prime}$, $\tau_{y z \text { out }}^{\prime}, \gamma_{x z, \text { out }}^{\prime}, \gamma_{y z \text { out }}^{\prime}$ in $S^{-}$, respectively. From the generalized Hooke's law, it follows that

$$
\begin{aligned}
\tau_{x z}^{0}+\tau_{x z, \text { in }}^{\prime} & =G^{\prime}\left(\gamma_{x z}^{0}+\gamma_{x z \text {,in }}^{\prime}\right) \\
& =\frac{G^{\prime}}{G} q_{1}+G^{\prime} \gamma_{x z \text {,in }}^{\prime} \text { in } S^{+} \\
\tau_{y z}^{0}+\tau_{y z, \text { in }}^{\prime} & =G^{\prime}\left(\gamma_{y z}^{0}+\gamma_{y z, \text { in }}^{\prime}\right) \\
& =\frac{G^{\prime}}{G} q_{2}+G^{\prime} \gamma_{y z \text {,in }}^{\prime} \text { in } S^{+} \\
\tau_{x z}^{0}+\tau_{x z, \text { out }}^{\prime} & =G\left(\gamma_{x z}^{0}+\gamma_{x z, \text { out }}^{\prime}\right) \\
& =q_{1}+G \gamma_{x z, \text { out }}^{\prime} \text { in } S^{-}
\end{aligned}
$$

$$
\begin{aligned}
\tau_{y z}^{0}+\tau_{y z \text { out }}^{\prime} & =G\left(\gamma_{y z}^{0}+\gamma_{y z, \text { out }}^{\prime}\right) \\
& =q_{2}+G \gamma_{y z, \text { out }}^{\prime} \text { in } S^{-}
\end{aligned}
$$

Again consider the infinite homogeneous linearly elastic solid with the longitudinal shear modulus $G$ everywhere, including in $S^{+}$. Instead of dealing with the presence of actual doubly periodic inclusions with a different elasticity, it is convenient and effective to introduce a suitable eigenstrain field $\left(\gamma_{x z}^{\prime \prime}, \gamma_{y z}^{\prime \prime}\right)$ in $S^{+}$, such that the equivalent homogeneous solid has the same strain and stress fields as the actual doubly periodic heterogeneous solid under the applied uniform shear stress at infinity, whichever may be the case. The introduction of the eigenstrain disturbs the uniform stress and strain fields, with the disturbance stress, and strain fields being denoted by $\tau_{x z, \text { in }}^{\prime \prime \prime}, \quad \tau_{y z \text {,in }}^{\prime \prime \prime}, \quad \gamma_{x z, \text { in }}^{\prime \prime \prime}=\tau_{x z, \text { in }}^{\prime \prime \prime} / G$, $\gamma_{y z, \text { in }}^{\prime \prime \prime}=\tau_{y z \text { in }}^{\prime \prime \prime} / G$ in $S^{+} ; \tau_{x z, \text { out }}^{\prime \prime \prime}, \tau_{y z, \text { out }}^{\prime \prime \prime}, \gamma_{x z, \text { out }}^{\prime \prime \prime}=\tau_{x z, \text { out }}^{\prime \prime \prime} / G$, $\gamma_{y z \text { out }}^{\prime \prime \prime \prime}=\tau_{y z \text {,out }}^{\prime \prime \prime} / G$ in $S^{-}$, respectively. Thus, the resulting variable strain and stress fields are, respectively, $\gamma_{x z}^{0}+\gamma_{x z}^{\prime \prime}+\gamma_{x z, \text { in }}^{\prime \prime \prime}, \quad \gamma_{y z}^{0}+\gamma_{y z}^{\prime \prime}+\gamma_{y z \text {,in }}^{\prime \prime \prime}, \quad \tau_{x z}^{0}+$ $\tau_{x z \text {,in }}^{\prime \prime \prime}$ and $\tau_{y z}^{0}+\tau_{y z \text {,in }}^{\prime \prime \prime}$ in the inclusion region $S^{+}$; $\gamma_{x z}^{0}+\gamma_{x z, \text { out }}^{\prime \prime \prime}, \gamma_{y z}^{0}+\gamma_{y z, \text { out }}^{\prime \prime \prime}, \tau_{x z}^{0}+\tau_{x z \text { out }}^{\prime \prime \prime}$ and $\tau_{y z}^{0}+\tau_{y z, \text { out }}^{\prime \prime \prime}$ in the matrix region $S^{-}$. From the generalized Hooke's law, it follows that

$$
\begin{aligned}
\tau_{x z}^{0}+\tau_{x z, \text { in }}^{\prime \prime \prime} & =G\left(\frac{q_{1}}{G}+\gamma_{x z}^{\prime \prime}+\gamma_{x z, \text { in }}^{\prime \prime \prime}-\gamma_{x z}^{\prime \prime}\right) \\
& =q_{1}+G \gamma_{x z, \text { in }}^{\prime \prime \prime} \quad \text { in } S^{+} \\
\tau_{y z}^{0}+\tau_{y z \text { in }}^{\prime \prime \prime} & =G\left(\frac{q_{2}}{G}+\gamma_{y z}^{\prime \prime}+\gamma_{y z, \text { in }}^{\prime \prime \prime}-\gamma_{y z}^{\prime \prime}\right) \\
& =q_{2}+G \gamma_{y z, \text { in }}^{\prime \prime \prime} \quad \text { in } S^{+} \\
\tau_{x z}^{0}+\tau_{x z, \text { out }}^{\prime \prime \prime} & =G\left(\frac{q_{1}}{G}+\gamma_{x z, \text { out }}^{\prime \prime \prime}\right) \\
& =q_{1}+G \gamma_{x z, \text { out }}^{\prime \prime \prime} \quad \text { in } S^{-} \\
\tau_{y z}^{0}+\tau_{y z, \text { out }}^{\prime \prime \prime} & =G\left(\frac{q_{2}}{G}+\gamma_{z z, \text { out }}^{\prime \prime \prime}\right) \\
& =q_{2}+G \gamma_{y z, \text { out }}^{\prime \prime \prime} \quad \text { in } S^{-}
\end{aligned}
$$

The so called consistency conditions require that the resulting stress field in the equivalent homogeneous solid is the same as that in the actual heterogeneous solid under the applied overall loads. From Eqs. (1), (2) and Eqs. (5), (6), it follows that 


$$
\begin{aligned}
& G^{\prime}\left(\frac{q_{1}}{G}+\gamma_{x z}^{\prime \prime}+\gamma_{x z, \text { in }}^{\prime \prime \prime}\right)=G\left(\frac{q_{1}}{G}+\gamma_{x z, \text { in }}^{\prime \prime \prime}\right) \text { in } S^{+} \\
& G^{\prime}\left(\frac{q_{2}}{G}+\gamma_{y z}^{\prime \prime}+\gamma_{y z, \text { in }}^{\prime \prime \prime}\right)=G\left(\frac{q_{2}}{G}+\gamma_{y z, \text { in }}^{\prime \prime \prime}\right) \text { in } S^{+}
\end{aligned}
$$

Eqs. (9) and (10) contain four unknown strain components $\gamma_{x z}^{\prime \prime}, \gamma_{y z}^{\prime \prime}, \gamma_{x z \text {,in }}^{\prime \prime \prime}$ and $\gamma_{y z \text {,in }}^{\prime \prime \prime}$. Their complete determination requires two additional equations relating the eigenstrain components $\gamma_{x z}^{\prime \prime}, \gamma_{z z}^{\prime \prime}$ with the corresponding disturbance strain components $\gamma_{x z, \text { in }}^{\prime \prime \prime}, \gamma_{y z, \text { in }}^{\prime \prime \prime}$, which results in a doubly quasi-periodic Riemann boundary problem.

\section{Doubly quasi-periodic Riemann boundary pro- blem}

It is well known that an arbitrary analytical function (complex potential) $f(z)$ can be used to formulate the longitudinal shear problem:

$w=f(z)+\overline{f(z)}$

$\tau_{x z}-\mathrm{i} \tau_{y z}=G\left(\gamma_{x z}-\mathrm{i} \gamma_{y z}\right)=2 G \frac{\mathrm{d} f(z)}{\mathrm{d} z}$

$T=\int_{A}^{B}\left(\tau_{x z} \mathrm{~d} y-\tau_{y z} \mathrm{~d} x\right)=G \mathrm{i}[\overline{f(z)}-f(z)]_{A}^{B}$

where $w$ is the longitudinal displacement, $G$ is the shear modulus, $\tau_{x z}, \tau_{y z}$ and $\gamma_{x z}, \gamma_{y z}$ are the shear stress and shear strain components, respectively, $T$ is the resultant shear stress on any arc $A B,[\bullet]_{A}^{B}$ signifies the change in the bracketed function in moving from point $A$ to point $B$ along any arc $A B$, and the overbar denotes the conjugate.

The eigenstrain in $S^{+}$in the equivalent homogeneous solid is doubly periodic, hence the corresponding disturbance stress and strain fields are also doubly periodic and the disturbance displacement field and the function $f(z)$ are doubly quasi-periodic (Lu, 1993; Li, 1999). Let $w_{0}(z)$ denote the displacement field corresponding to the eigenstain $\left(\gamma_{x z}^{\prime \prime}, \gamma_{y z}^{\prime \prime}\right)$, which is not related to the stress field, but can formally be expressed by a complex function $f_{0}(z)$ in $S_{0}^{+}$in $P_{00}$. Expand $f_{0}(z)$ to a Taylor series in $S_{0}^{+}$: $f_{0}(z)=\sum_{k=1}^{\infty} A_{k} z^{k} \quad z \in S_{0}^{+}$

Then from Eq. (11), it follows that

$$
\begin{aligned}
w_{0}(t) & =\sum_{k=1}^{\infty} A_{k} t^{k}+\sum_{k=1}^{\infty} \bar{A}_{k} \bar{t}_{k} \\
& =\sum_{k=1}^{\infty} A_{k} t^{k}+\sum_{k=1}^{\infty} \bar{A}_{k} R^{2 k} \frac{1}{t^{k}} \quad t \in L_{0}
\end{aligned}
$$

where $R$ is the radius of the contour $L_{0}$, and the term corresponding to the rigid-body displacement has been ignored (otherwise, a trivial coordinate transformation can be introduced). From Eq. (12), it follows that

$\gamma_{x z}^{\prime \prime}-\mathrm{i} \gamma_{y z}^{\prime \prime}=2 \frac{\mathrm{d} f_{0}(z)}{\mathrm{d} z}=2 \sum_{k=1}^{\infty} k A_{k} z^{k-1} \quad z \in S_{0}^{+}$

From the definition of the eigenstrain, the conditions of the disturbance displacement jump and the disturbance stress continuity on $L=L_{0}+\Omega_{m n}$, where $\Omega_{m n}=2 m \omega_{1}+2 n \omega_{2}(m, n=0, \pm 1, \pm 2, \ldots)$, can be written as

$$
\begin{aligned}
& w^{+}(t)+w_{0}(t)=w^{-}(t) \quad t \in L_{0}+\Omega_{m n} \\
& T^{+}(t)=T^{-}(t) \quad t \in L_{0}+\Omega_{m n}
\end{aligned}
$$

where the superscripts + and - signify the function value as approached from $S^{+}$and $S^{-}$, respectively. The substitution of Eq. (11) into the disturbance displacement jump condition (17) yields

$$
\begin{aligned}
& {\left[f^{+}(t)+\overline{f^{+}(t)}\right]-\left[f^{-}(t)+\overline{f^{-}(t)}\right]=-w_{0}(t)} \\
& \quad t \in L_{0}+\Omega_{m n}
\end{aligned}
$$

and the substitution of Eq. (12) into the disturbance stress continuity condition (18) yields

$$
\begin{aligned}
& {\left[f^{+}(t)-\overline{f^{+}(t)}\right]-\left[f^{-}(t)-\overline{f^{-}(t)}\right]=0} \\
& \quad t \in L_{0}+\Omega_{m n}
\end{aligned}
$$

For eigenstrain problems, no loads at infinity are applied. According to the periodicity, the resultant stress on each boundary $\Gamma_{k}(k=1,2,3,4$; see Fig. 1 ) of the fundamental cell $P_{00}$ vanishes. Thus, from Eq. (13), it follows that

$$
[f(z)-\overline{f(z)}]_{\Gamma_{k}}=0 \quad k=1,2
$$


The addition of Eqs. (19) and (20) yields

$$
\begin{aligned}
& f^{+}(t)-f^{-}(t)=-\frac{1}{2} w_{0}(t)=g(t) \\
& \quad t \in L_{0}+\Omega_{m n}
\end{aligned}
$$

Substituting Eq. (28) into Eq. (23) and integrating, then $f(z)$ in the fundamental cell $P_{00}$ can be expressed as

$f(z)= \begin{cases}C_{1} z-\frac{1}{2}\left[\sum_{k=1}^{\infty} A_{k} z^{k}+\sum_{k=1}^{\infty} \frac{(-1)^{k}}{(k-1) !} \bar{A}_{k} R^{2 k}\left(\zeta(z)-\frac{1}{z}\right)^{(k-1)}\right] & z \in S_{0}^{+} \\ C_{1} z-\frac{1}{2} \sum_{k=1}^{\infty} \frac{(-1)^{k}}{(k-1) !} \bar{A}_{k} R^{2 k} \zeta^{(k-1)}(z) & z \in S_{0}^{-}\end{cases}$

According to the new results for doubly quasiperiodic Riemann boundary value problems $(\mathrm{Lu}$, 1993), the solution of Eq. (22) can be expressed as

$f(z)=C_{0}+C_{1} z+\frac{1}{2 \pi \mathrm{i}} \int_{L_{0}} g(t) \zeta(t-z) \mathrm{d} t$

where $\zeta\left(\right.$ ) is the Weierstrass Zeta function, $C_{0}$ is a constant representing the rigid displacement and can be ignored, the complex constant $C_{1}$ can be determined by the resultant stress condition on the fundamental cell boundary cell. The substitution of Eq. (23) into Eq. (21) yields

$2 \omega_{k} C_{1}-2 \bar{\omega}_{k} \bar{C}_{1}=\frac{\eta_{k}}{\pi \mathrm{i}} \int_{L_{0}} g(t) \mathrm{d} t+\frac{\bar{\eta}_{k}}{\pi \mathrm{i}} \int_{L_{0}} \overline{g(t) \mathrm{d} t}$

from which

$C_{1}=-\frac{\mathrm{i}}{2 S}\left[\delta_{2} \int_{L_{0}} g(t) \mathrm{d} t+\int_{L_{0}} \overline{g(t) \mathrm{d} t}\right]$

where $S$ is the area of the fundamental cell $P_{00}$

$S=2 \mathrm{i}\left(\omega_{1} \bar{\omega}_{2}-\omega_{2} \bar{\omega}_{1}\right)$

and

$\delta_{2}=\frac{2}{\pi \mathrm{i}}\left(\bar{\omega}_{1} \eta_{2}-\bar{\omega}_{2} \eta_{1}\right)$

where $\eta_{k}=\zeta\left(\omega_{k}\right) k=1,2$.

From Eq. (15) and (22), it is seen that

$$
\begin{aligned}
g(t) & =-\frac{1}{2} w_{0}(t) \\
& =-\frac{1}{2}\left(\sum_{k=1}^{\infty} A_{k} t^{k}+\sum_{k=1}^{\infty} \bar{A}_{k} R^{2 k} \frac{1}{t^{k}}\right) \quad t \in L_{0}
\end{aligned}
$$

where the superscript $k-1$ denotes the derivative of order $k-1$, and the constant $C_{0}$ representing the rigid-body displacement has been ignored.

Substituting Eq. (28) into Eq. (25) and using the residue theorem, the complex constant $C_{1}$ is related to $A_{1}$ :

$C_{1}=\frac{\pi R^{2}}{2 S}\left(A_{1}-\delta_{2} \bar{A}_{1}\right)$

\section{Solution}

Noting that the complex potential (29) is corresponding to the fields disturbed by eigenstrain, from Eq. (12), it is seen that

$\gamma_{x z}^{\prime \prime \prime}-\mathrm{i} \gamma_{y z}^{\prime \prime \prime}=2 \frac{\mathrm{d}}{\mathrm{d} z} f(z)$

The substitution of Eq. (29) into Eq. (31) yields

$$
\begin{aligned}
\gamma_{x z, \text { in }}^{\prime \prime \prime}-\mathrm{i} \gamma_{z z, \text { in }}^{\prime \prime \prime}= & 2 C_{1}-\left[\sum_{k=1}^{\infty} k A_{k} z^{k-1}+\sum_{k=1}^{\infty} \frac{(-1)^{k}}{(k-1) !}\right. \\
& \left.\times \bar{A}_{k} R^{2 k}\left(\zeta(z)-\frac{1}{z}\right)^{(k)}\right] \quad z \in S_{0}^{+}
\end{aligned}
$$

$$
\begin{aligned}
& \gamma_{x z, \text { out }}^{\prime \prime \prime}-\mathrm{i} \gamma_{y z, \text { out }}^{\prime \prime \prime}=2 C_{1}-\sum_{k=1}^{\infty} \frac{(-1)^{k}}{(k-1) !} \bar{A}_{k} R^{2 k} \zeta^{(k)}(z) \\
& z \in S_{0}^{-}
\end{aligned}
$$


Eqs. (9) and (10) can cast into complex form:

$$
\begin{aligned}
& \left(\frac{G^{\prime}}{G}-1\right)\left(q_{1}-\mathrm{i} q_{2}\right)+\left(G^{\prime}-G\right)\left(\gamma_{x z, \text { in }}^{\prime \prime \prime}-\mathrm{i} \gamma_{y z, \text { in }}^{\prime \prime \prime}\right) \\
& \quad+G^{\prime}\left(\gamma_{x z}^{\prime \prime}-\mathrm{i} \gamma_{y z}^{\prime \prime}\right)=0 \quad \text { in } S^{+}
\end{aligned}
$$

The substitution of Eqs. (16) and (32) into Eq. (34) yields

$$
\begin{aligned}
& \left(\frac{G^{\prime}}{G}-1\right)\left(q_{1}-\mathrm{i} q_{2}\right)+\left(G^{\prime}-G\right)\left\{\frac{\pi R^{2}}{S}\left(A_{1}-\delta_{2} \bar{A}_{1}\right)\right. \\
& \left.-\sum_{k=1}^{\infty} k A_{k} z^{k-1}-\sum_{k=1}^{\infty} \frac{(-1)^{k}}{(k-1) !} \bar{A}_{k} R^{2 k}\left[\zeta(z)-\frac{1}{z}\right]^{(k)}\right\} \\
& +2 G^{\prime} \sum_{k=1}^{\infty} k A_{k} z^{k-1}=0
\end{aligned}
$$

The complex constants $A_{k}$ are determined by comparing the coefficients in the same order terms, and the problem is solved.

In the actual heterogeneous material and homogeneous equivalent solid, the stress fields are the same. From Eqs. (1)-(8), (16), (32) and (33), the stress fields in the inclusion $\left(S_{0}^{+}\right)$and matrix $\left(S_{0}^{-}\right)$can be expressed as

$$
\begin{aligned}
\tau_{x z}-\mathrm{i} \tau_{y z}= & \frac{G^{\prime}}{G}\left(q_{1}-\mathrm{i} q_{2}\right)+G^{\prime}\left[\left(\gamma_{x z}^{\prime \prime}+\gamma_{x z, \text { in }}^{\prime \prime \prime}\right)-\mathrm{i}\left(\gamma_{y z}^{\prime \prime}+\gamma_{y z, \text { in }}^{\prime \prime \prime}\right)\right] \\
= & \frac{G^{\prime}}{G}\left(q_{1}-\mathrm{i} q_{2}\right)+G^{\prime}\left\{\frac{\pi R^{2}}{S}\left(A_{1}-\delta_{2} \bar{A}_{1}\right)\right. \\
& +\sum_{k=1}^{\infty} k A_{k} z^{k-1}-\sum_{k=1}^{\infty} \frac{(-1)^{k}}{(k-1) !} \\
& \left.\times \bar{A}_{k} R^{2 k}\left[\zeta(z)-\frac{1}{z}\right]^{(k)}\right\} z \in S_{0}^{+}
\end{aligned}
$$

and

$$
\begin{aligned}
\tau_{x z}-\mathrm{i} \tau_{y z}= & q_{1}-\mathrm{i} q_{2}+G\left(\gamma_{x z, \text { out }}^{\prime \prime \prime}-\mathrm{i} \gamma_{y z, \text { out }}^{\prime \prime \prime}\right) \\
= & q_{1}-\mathrm{i} q_{2}+G\left[\frac{\pi R^{2}}{S}\left(A_{1}-\delta_{2} \bar{A}_{1}\right)\right. \\
& \left.-\sum_{k=1}^{\infty} \frac{(-1)^{k}}{(k-1) !} \bar{A}_{k} R^{2 k} \zeta^{(k)}(z)\right] \quad z \in S_{0}^{-}
\end{aligned}
$$

As the special cases of the present solution, several problems of practical importance are solved or resolved.
(1) A single inclusion in an infinite homogeneous medium under longitudinal shear at infinity. Letting $q_{1}=q, q_{2}=0,\left|\omega_{1}\right| \rightarrow \infty,\left|\omega_{2}\right| \rightarrow \infty$, then $S \rightarrow \infty$ and $\zeta(z)$ degenerates into

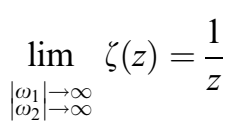

Hence, the Eq. (35) degenerates into

$$
\left(\frac{G^{\prime}}{G}-1\right) q+\left(G^{\prime}+G\right) \sum_{k=1}^{\infty} k A_{k} z^{k-1}=0
$$

from which

$$
A_{1}=-\frac{G^{\prime}-G}{G\left(G^{\prime}+G\right)} q \quad A_{k}=0 \quad k=2,3, \ldots
$$

The substitution of Eq. (40) into Eqs. (36) and (37) yields the solutions in the inclusion $\left(S^{+}\right)$ and matrix $\left(S^{-}\right)$:

$\tau_{x z}-\mathrm{i} \tau_{y z}=\frac{2 G^{\prime}}{G^{\prime}+G} q$ in $S^{+}$

and

$\tau_{x z}-\mathrm{i} \tau_{y z}=q+\frac{G^{\prime}-G}{G^{\prime}+G} q R^{2} \frac{1}{z^{2}} \quad$ in $S^{-}$

The solutions (41) and (42) are in agreement with the classical results for a single inclusion in an infinite homogeneous medium under longitudinal shear at infinity (for example, see Jiang and Cheung, 2001).

(2) A single-row periodic inclusion in an infinite matrix. Letting $\left|\omega_{1}\right| \rightarrow a \pi,\left|\omega_{2}\right| \rightarrow \infty$, then the function $\zeta(z)$ degenerates into

$$
\lim _{\substack{\omega_{1}\left|\rightarrow a \pi \\ \omega_{2}\right| \rightarrow \infty}} \zeta(z)=\frac{1}{3 a^{2}}+\frac{1}{a} \operatorname{ctg}\left(\frac{z}{a}\right)
$$

The solution is arrived by substituting Eq. (43) into Eqs. (35)-(37).

(3) Doubly periodic holes or doubly periodic rigid inclusions. The problem is solved as the special case of the inclusion modulus $G^{\prime}=0$ or $G^{\prime} \rightarrow \infty$.

Finally examine a special case that the modulus of the doubly periodic inclusions is equal to that of 
the matrix. Letting $G^{\prime}=G$, then from Eq. (35) it follows that $A_{k}=0(k=1,2,3, \ldots)$. Eqs. (36) and (37) both become $\tau_{x z}-\mathrm{i} \tau_{y z}=q$, and obviously the result is expected.

\section{Numerical examples and discussions}

In this section, illustrative examples are given to demonstrate the application of the solution developed above in analysis of heterogeneous materials. The computational object is shown in Fig. 2, where the fundamental cell is a parallelogram with the side lengths $2 a_{1}$ and $2 a_{2}$ and a vertex angle $\theta$. The fundamental periods can be expressed as

$2 \omega_{1}=2 a_{1}, \quad 2 \omega_{2}=2 a_{2}(\cos \theta+\mathrm{i} \sin \theta)$

The overall elastic properties and local stress concentrations are of great concerns in mechanics of heterogeneous materials. For an anisotropic heterogeneous material or a composite material, the effective compliance tensor $\overline{\mathbf{D}}$ can be determined by the following equation (Nemat-Nasser and Hori, 1999):

$$
(\mathbf{D}-\overline{\mathbf{D}}): \boldsymbol{\sigma}^{0}=\sum_{\alpha=1}^{n} \lambda_{\alpha}\left(\mathbf{D}-\mathbf{D}^{\alpha}\right): \overline{\boldsymbol{\sigma}}^{\alpha}
$$

where $\mathbf{D}$ and $\mathbf{D}^{\alpha}$ are the compliance tensors of the matrix and $\alpha$ th reinforcement, respectively; $\boldsymbol{\sigma}^{0}$ is the stress tensor at the far-field; $\overline{\boldsymbol{\sigma}}^{\alpha}$ is the average stress tensor of the $\alpha$ th reinforcement; $\lambda_{\alpha}$ is the volume fraction of the $\alpha$ th reinforcement.

For a fiber reinforced composite with isotropic fiber and matrix materials, the equation to determine the effective longitudinal moduli can be obtained from a degenerate case of Eq. (45):

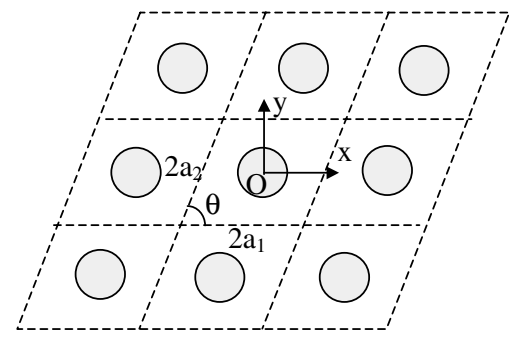

Fig. 2. Computational object: an infinite solid with an a parallelogrammic array of cylindrical inclusions.

$$
\begin{aligned}
& {\left[\begin{array}{cc}
-\frac{1}{G}-\bar{D}_{44} & -\bar{D}_{45} \\
-\bar{D}_{45} & \frac{1}{G}-\bar{D}_{55}
\end{array}\right]\left\{\begin{array}{l}
\tau_{y z}^{\infty} \\
\tau_{x z}^{\infty}
\end{array}\right\}} \\
& =\lambda\left[\begin{array}{cc}
\frac{1}{G}-\frac{1}{G^{\prime}} & 0 \\
0 & \frac{1}{G}-\frac{1}{G^{\prime}}
\end{array}\right]\left\{\begin{array}{l}
\bar{\tau}_{y z, f} \\
\bar{\tau}_{x z, f}
\end{array}\right\}
\end{aligned}
$$

where $G^{\prime}$ and $G$ are the shear moduli of fiber and matrix materials, respectively; $\tau_{y z}^{\infty}$ and $\tau_{x z}^{\infty}$ are the longitudinal shear stress components at the farfield; $\bar{\tau}_{y z, f}$ and $\bar{\tau}_{x z, f}$ are the average longitudinal shear stress component in the fibers; $\lambda$ is the fiber volume fraction:

$\lambda=\frac{\pi R^{2}}{4 a_{1} a_{2} \sin \theta}$

where $R$ is the fiber radius.

Once $\bar{\tau}_{y z \mathrm{f}}$ and $\bar{\tau}_{x z \mathrm{f}}$ are determined by the method developed in this paper, the effective longitudinal compliance components $\bar{D}_{44}, \bar{D}_{45}$ and $\bar{D}_{55}$ can be obtained by Eq. (46). Then the inverse of effective compliance matrix yields the effective longitudinal shear modulus matrix:

$\left[\begin{array}{ll}\bar{C}_{44} & \bar{C}_{45} \\ \bar{C}_{45} & \bar{C}_{55}\end{array}\right]=\left[\begin{array}{ll}\bar{D}_{44} & \bar{D}_{45} \\ \bar{D}_{45} & \bar{D}_{55}\end{array}\right]^{-1}$

The presence of inclusions leads to local stress concentrations. The stress field within the inclusion is uniform for a single ellipsoidal inclusion in an infinite homogeneous linearly elastic solid under uniform stress state at infinity (Eshelby, 1957). Even for a single circular or elliptic inclusion with certain coating (three phase model), the uniformity of the inclusion stress field may also be observed (for example, see Jiang and Cheung, 1998, 2001; Jiang et al., 2001). However, the interaction among multiple inclusions would violate the uniformity of inclusion stress field (refer to Fig. 3 in Example 1).

Without loss of generality, letting

$\tau_{x z}^{\infty}=q_{1}=q, \quad \tau_{y z}^{\infty}=q_{2}=0$

$\left(q_{2}=0\right.$ can always be arrived at by using a proper rotation of coordinates), then a stress concentration factor $\beta$ can be defined as

$\beta=\tau_{x z, \max } / q$

where $\tau_{x z \text { max }}$ is the maximum value of $\tau_{x z}$. 
In the following numerical computations, the emphasis is on the influence of inclusion array on the overall elastic properties and the local stress concentrations.

\section{Example 1. Square array}

This is a special case of the parallelogrammic array, i.e., $a_{1}=a_{2}=a$ and $\theta=90^{\circ}$. First examine the disturbance of the inclusion (fiber) stress field under interaction of multiple inclusions, where the far-field stress is prescribed by Eq. (49). The distribution of the dimensionless shear stress component $\tau_{x z} / q$ in the fiber for $R / a=0.5,0.9$ is plotted in Fig. 3. Owing to the symmetry/antisymmetry it is sufficient to show only the onequarter of the inclusion.

It is observed from Fig. 3 that the stress nonuniformity increases with a increase of the dimensionless fiber radius $R / a$ while holding inclusion/matrix modulus ratio $G^{\prime} / G$ fixed. Numerical results show also that the non-uniformity of the stress distribution increases with a increase of $G^{\prime} / G$ while holding the dimensionless fiber radius fixed. In addition, the inclusion/ matrix modulus dismatch also leads to a shear stress component $\tau_{y z}$ in the $y$-direction (perpendicular to the load direction), which is small and is not plotted in the figure. In spite of a serious disturbance of the stress, the numerical results show that the averaged stress in the fundamental cell remains accurately unchanged, i.e., $\bar{\tau}_{x z}=q$, $\bar{\tau}_{y z}=0$.
In the case of the square array, the composite material is transversely isotropic, and we have $\bar{C}_{44}=\bar{C}_{55}=G_{\mathrm{e}}, \bar{C}_{45}=0$.

The problem of the square array was studied by other methods, such as the finite difference method (Adam and Doner, 1967) and Fourier method (Chen, 1970). A comparison of the results by the three methods (the present, Fourier, and finite difference methods) is shown in Table 1.

It is seen from Table 1 that in previous studies, the Fourier method and the finite difference method show good agreement for the effective longitudinal shear modulus but poor agreement for the stress concentration factor. The previous investigator (Chen, 1970) was not sure which is more accurate and he supposed that the finite difference method probably gave a more accurate prediction of the stress concentration factor. The present method is a rigorous analytical method and can be used to estimate the accuracy of other numerical and approximate methods. It is concluded from Table 1 that both the Fourier and finite difference methods gave accurate predictions for the effective longitudinal shear modulus, whereas in this example, the Fourier method is more accurate for the stress concentration factor than the finite difference methods.

Example 2. Hexagonal array and a comparison with the square array

In this case, $a_{1}=a_{2}=a$ and $\theta=60^{\circ}$, and Eq. (44) (fundamental periods) becomes
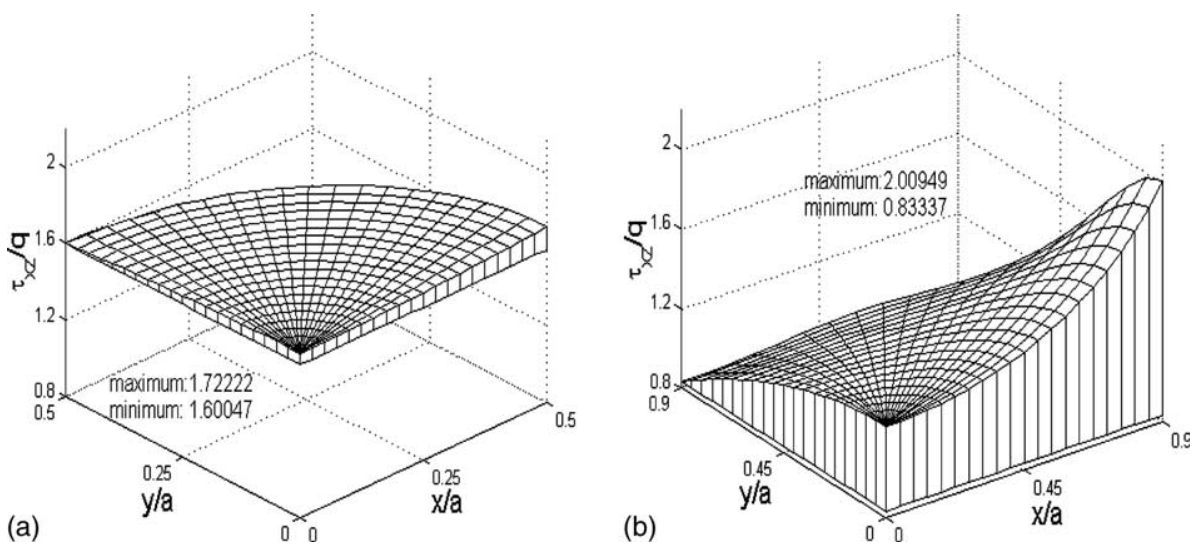

Fig. 3. Distribution of the dimensionless shear stress $\tau_{x z} / q$ in fiber $\left(G^{\prime} / G=100\right)$. 
Table 1

A comparison of the numerical results by three different methods

\begin{tabular}{|c|c|c|c|c|c|c|c|}
\hline \multirow[t]{2}{*}{$\lambda$} & \multirow[t]{2}{*}{$G^{\prime} / G$} & \multicolumn{3}{|l|}{$\beta$} & \multicolumn{3}{|l|}{$G_{\mathrm{e}} / G$} \\
\hline & & Present & $\mathrm{FM}^{\mathrm{a}}$ & $\mathrm{DM}^{\mathrm{b}}$ & Present & FM & DM \\
\hline \multirow[t]{4}{*}{0.4} & 6 & 1.49610 & 1.496 & 1.849 & 1.80451 & 1.805 & 1.796 \\
\hline & 20 & 1.61796 & 1.618 & 2.110 & 2.14586 & 2.147 & 2.137 \\
\hline & 120 & 1.66757 & 1.668 & 2.222 & 2.31343 & 2.314 & 2.305 \\
\hline & 400 & 1.67481 & 1.675 & - & 2.33957 & 2.340 & - \\
\hline \multirow[t]{4}{*}{0.55} & 6 & 1.56090 & 1.561 & 1.684 & 2.32562 & 2.326 & 2.304 \\
\hline & 20 & 1.72279 & 1.723 & 1.906 & 3.07712 & 3.184 & 3.044 \\
\hline & 120 & 1.79212 & 1.792 & 2.006 & 3.50663 & 3.555 & 3.469 \\
\hline & 400 & 1.80241 & 1.802 & - & 3.57789 & 3.578 & - \\
\hline \multirow[t]{4}{*}{0.7} & 6 & 1.85000 & 1.851 & 2.152 & 3.17311 & 3.176 & 3.163 \\
\hline & 20 & 2.22401 & 2.226 & 2.829 & 5.21367 & 5.222 & 5.187 \\
\hline & 120 & 2.41071 & 2.413 & 3.204 & 6.92945 & 6.945 & 6.878 \\
\hline & 400 & 2.44001 & 2.443 & - & 7.27364 & 7.291 & - \\
\hline \multirow[t]{4}{*}{0.75} & 6 & 2.15701 & 2.156 & 2.837 & 3.61966 & 3.620 & 3.646 \\
\hline & 20 & 2.90501 & 2.904 & 4.618 & 7.00409 & 7.006 & 7.005 \\
\hline & 120 & 3.35996 & 3.354 & 5.851 & 11.1640 & 11.170 & 11.035 \\
\hline & 400 & 3.44510 & 3.490 & - & 12.2264 & 12.523 & - \\
\hline
\end{tabular}

${ }^{\mathrm{a}}$ FM- the Fourier method (Chen, 1970).

${ }^{\mathrm{b}} \mathrm{DM}$ - the finite difference method (Adam and Doner, 1967).

$2 \omega_{1}=2 a, \quad 2 \omega_{2}=(1+\mathrm{i} \sqrt{3}) a$

The numerical results show that a composite material with a hexagonal array of fibers is still transversely isotropic. A comparison of the dimensionless effective longitudinal shear modulus for the hexagonal and square arrays is shown in Table 2. It is observed that the hexagonal array always yields a lower value of the effective modulus than the square array when the volume fraction $\lambda$ is the same.

The interfacial shear stress plays an important role in fiber debonding. To examine such a stress, introduce polar coordinates $r, \theta$ as shown in Fig. 4. Under the far-field stress prescribed by Eq. (49), the distribution of the dimensionless interfacial longitudinal shear stress $\tau_{r z} / q$ is shown in Fig. 5 for square and hexagonal arrays of fibers. Owing to the symmetry/antisymmetry, the stress distribution is plotted only on the one-quarter portion of the fiber/matrix interface.

It is observed that when $\lambda$ is small, the distributions of the interfacial shear stress for the two arrays are almost the same and both of them approach to that for a single fiber in an infinite matrix. For a large value of $\lambda$, their difference becomes remarkable. The maximum value of $\tau_{r z} / q$ for the two arrays occurs at $\theta^{\prime}=0$, and the hexagonal array gives rise to a lower maximum value than the square array. The fact indicates that the hexagonal array is more reasonable from the strength view.

Example 3. Rectangular array

In this case $\theta=90^{\circ}$ and Eq. (44) (fundamental periods) becomes

Table 2

A comparison of $G_{\mathrm{e}} / G$ for the two fiber arrays $\left(G^{\prime} / G=120\right)$

\begin{tabular}{lllllll}
\hline$\lambda$ & 0.1 & 0.2 & 0.3 & 0.4 & 0.5 & 0.6 \\
\hline Hexagonal & 1.21815 & 1.48971 & 1.83711 & 2.29764 & 2.93931 & 3.90319 \\
Square & 1.21816 & 1.49000 & 1.83990 & 2.31343 & 3.00863 & 4.18637 \\
\hline
\end{tabular}




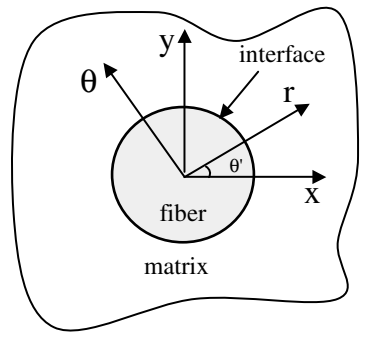

Fig. 4. The polar coordinates $(r, \theta)$ with the origin at the center of a fiber section.

$2 \omega_{1}=2 a_{1}, \quad 2 \omega_{2}=2 \mathrm{i} a_{2}$

Under the far-field stress prescribed by Eq. (49), the variations of the stress concentration factor $\beta$ with the dimensionless fiber spacings $b_{1} / 2 R$ and $b_{2} / 2 R$ are listed in Table 3 , where $b_{1}=2\left(a_{1}-R\right)$ in

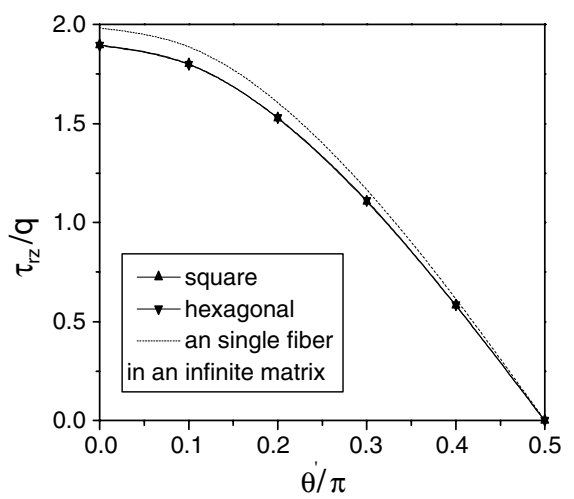

(a)

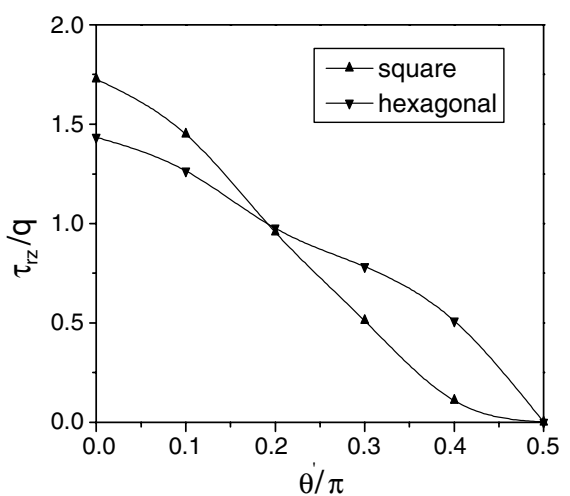

(c) the $x$-direction and $b_{2}=2\left(a_{2}-R\right)$ in the $y$-direction.

An interesting phenomenon is observed from Table 3. The stress concentration factor $\beta$ depends contrarily on the two dimensionless spacings, i.e., decreasing the dimensionless spacing $b_{1} / 2 R$, tends to increase the dimensionless stress concentration factor $\beta$; but decreasing $b_{2} / 2 R$ tends to decrease $\beta$. This fact strongly implies that in studying stress concentration of multiple inclusions, only an inclusion density parameter (such as the inclusion volume fraction) is insufficient, and other factor must be considered and extracted.

Example 4. Rhombic array

In this case, $a_{1}=a_{2}=a$, Eq. (44) (fundamental periods) becomes

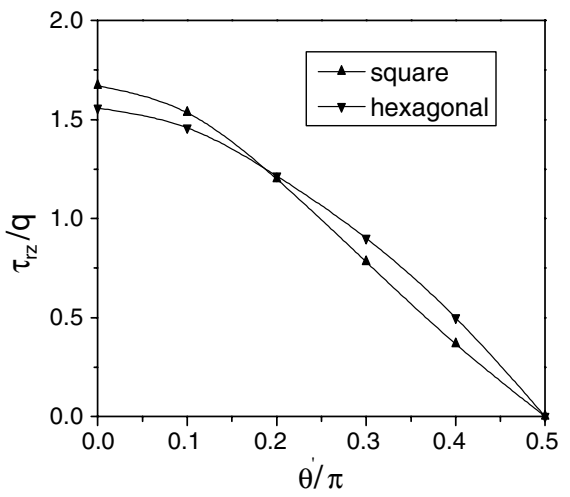

(b)

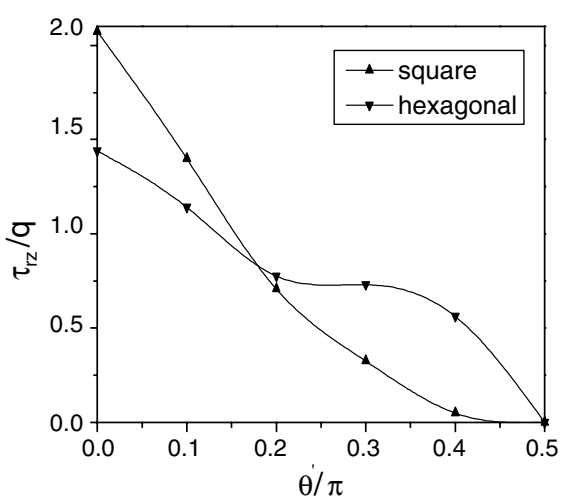

(d)

Fig. 5. Dimensionless interfacial longitudinal shear stress $\tau_{r z} / q$ for two fiber arrays $\left(G^{\prime} / G=120\right.$ ). (a) $\lambda=0.05$, (b) $\lambda=0.3$, (c) $\lambda=0.5$, (d) $\lambda=0.65$. 
Table 3

Variations of stress concentration factor $\beta$ with inclusion spacings $\left(G^{\prime} / G=100\right)$

\begin{tabular}{llllll}
\hline$b_{2} / 2 R$ & $\beta$ & & & \\
\cline { 2 - 5 } & $b_{1} / 2 R=0.1$ & $b_{1} / 2 R=0.3$ & $b_{1} / 2 R=0.5$ & $b_{1} / 2 R=0.7$ & $b_{1} / 2 R=1$ \\
\hline 0.1 & 2.06392 & 1.55996 & 1.46226 & 1.43396 & 1.42382 \\
0.5 & 2.57502 & 1.83162 & 1.66031 & 1.59857 & 1.56730 \\
1 & 3.16745 & 2.14406 & 1.88874 & 1.78511 & 1.72222 \\
\hline
\end{tabular}

Table 4

Effective elastic moduli $\bar{C}_{44} / G, \bar{C}_{55} / G, \bar{C}_{45} / G\left(G^{\prime} / G=120\right)$

\begin{tabular}{|c|c|c|c|c|c|c|}
\hline \multirow[t]{2}{*}{$\lambda$} & \multicolumn{3}{|l|}{$\theta=75^{\circ}$} & \multicolumn{3}{|l|}{$\theta=45^{\circ}$} \\
\hline & $\bar{C}_{44} / G$ & $\bar{C}_{45} / G$ & $\bar{C}_{55} / G$ & $\bar{C}_{44} / G$ & $\bar{C}_{45} / G$ & $\bar{C}_{55} / G$ \\
\hline 0.1 & 1.21780 & 0.00133 & 1.21852 & 1.22306 & -0.00471 & 1.21365 \\
\hline 0.2 & 1.48816 & 0.00672 & 1.49176 & 1.51608 & -0.02383 & 1.46841 \\
\hline 0.3 & 1.83374 & 0.01968 & 1.84428 & 1.92172 & -0.07065 & 1.78042 \\
\hline 0.4 & 2.29477 & 0.04739 & 2.32016 & 2.53343 & -0.17671 & 2.18002 \\
\hline 0.5 & 2.95203 & 0.10602 & 3.00884 & 3.62069 & -0.44144 & 2.73782 \\
\hline 0.6 & 4.00126 & 0.23812 & 4.12884 & - & - & - \\
\hline
\end{tabular}

$2 \omega_{1}=2 a, \quad 2 \omega_{2}=2 a(\cos \theta+\mathrm{i} \sin \theta)$

Examples 1 and 2 are two special cases of the rhombic array, where $\theta=90^{\circ}$ for a square array and $\theta=60^{\circ}$ for a hexagonal array. In these two special cases, the composite material is transversely isotropic. However, for a general array of fibers, the composite material would be anisotropic. The results of the dimensionless effective longitudinal elastic moduli $\bar{C}_{44} / G, \bar{C}_{55} / G$ and $\bar{C}_{45} / G$ are listed in Table 4 for a rhombic array with $\theta=75^{\circ}$ and $45^{\circ}$.

For an anisotropic material, the elastic moduli depend upon the orientation of the reference axes. Let $\theta=45^{\circ}$ and assume that the $x^{\prime} y^{\prime}$ axes are located along the diagonals of the fundamental rhombus as shown in Fig. 6.

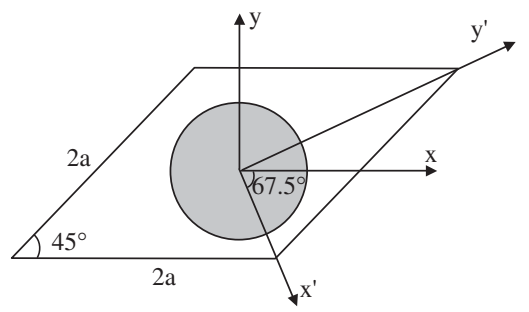

Fig. 6. $x y$ axes and $x^{\prime} y^{\prime}$ axes in the fundamental cell for a rhombic array.
The results of dimensionless effective elastic moduli $\bar{C}_{44} / G, \bar{C}_{55} / G$ and $\bar{C}_{45} / G$ with respect to the $x^{\prime} y^{\prime}$ axes are listed in Table 5 for such a rhombic array. It is seen that such a composite material is actually orthotropic and the principal axes lie along the diagonal of the fundamental rhombic.

The transformation equation for elastic moduli can be used to compute the elastic moduli with respect to arbitrary reference axes. Using the data in Table 5 and transformation equation for elastic moduli, the dimensionless effective elastic moduli $\bar{C}_{44} / G, \bar{C}_{55} / G$ and $\bar{C}_{45} / G$ with respect to $x y$ axes are computed and listed in Table 6 .

It is seen that the results in Table 6 are in exact agreement with those in Table 4 for $\theta=45^{\circ}$. The fact shows the accuracy of the present method. The rigorous analytical method developed in this paper can provide benchmark results for other numerical and approximate methods.

Example 5. Problems of doubly periodic voids and rigid inclusions

Letting the inclusion modulus $G^{\prime}=0$, the present method provides the results for solids with doubly periodic tunnel voids. A material weakened by square or hexagonal array of tunnel voids is transversely isotropic. The variations of the 
Table 5

Effective elastic moduli with respect to the $x^{\prime} y^{\prime}$ axes $\left(G^{\prime} / G=120, \theta=45^{\circ}\right)$

\begin{tabular}{llllll}
\hline$\lambda$ & 0.1 & 0.2 & 0.3 & 0.4 & 0.5 \\
\hline $\bar{C}_{44} / G$ & 1.21170 & 1.45854 & 1.75116 & 2.10682 & 2.55497 \\
$\bar{C}_{45} / G$ & 0 & 0 & 0 & 0 & 0 \\
$\bar{C}_{55} / G$ & 1.22501 & 1.52595 & 1.95099 & 2.60663 & 3.80354 \\
\hline
\end{tabular}

Table 6

Effective elastic moduli with respect to $x y$ axes $\left(G^{\prime} / G=120, \theta=45^{\circ}\right)^{\mathrm{a}}$

\begin{tabular}{lrrrrr}
\hline$\lambda$ & 0.1 & 0.2 & 0.3 & \multicolumn{1}{c}{0.4} & \multicolumn{1}{c}{0.5} \\
\hline $\bar{C}_{44} / G$ & 1.22306 & 1.51608 & 1.92172 & 2.53344 & 3.62069 \\
$\bar{C}_{45} / G$ & -0.00471 & -0.02383 & -0.07065 & -0.17671 & -0.44144 \\
$\bar{C}_{55} / G$ & 1.21365 & 1.46841 & 1.78042 & 2.18002 & 2.73782 \\
\hline
\end{tabular}

${ }^{\mathrm{a}}$ The results are obtained by a coordinate transform of the data in Table 5 .

Table 7

Variations of effective longitudinal shear modulus $G_{\mathrm{e}}$ with the void volume fraction $\lambda$ (matrix shear modulus $\left.G=30 \mathrm{GPa}\right)^{\mathrm{a}}$

\begin{tabular}{llllllll}
\hline$\lambda$ & 0 & 0.1 & 0.2 & 0.3 & 0.4 & 0.5 & 0.6 \\
\hline Hexagonal & 30.0000 & 24.5455 & 20.0000 & 16.1533 & 12.8534 & 9.9843 & 7.4503 \\
Square & 30.0000 & 24.5453 & 19.9959 & 16.1274 & 12.7605 & 9.7396 & 6.9096 \\
\hline
\end{tabular}

${ }^{\mathrm{a}}$ The effective longitudinal shear modulus cannot be directly computed by setting $G^{\prime}=0$. Instead, $G^{\prime}$ is taken as a small value approaching 0 , and in this example $G^{\prime}=10^{-6} \mathrm{GPa}$.

effective longitudinal shear modulus with the void volume fraction are shown in Table 7 , where the matrix shear modulus $G=30 \mathrm{GPa}$.

The effective longitudinal shear modulus of a solid with the voids in a hexagonal array is higher than that with voids in a square array when the void volume fraction is the same. This law is contrary to that for soft matrix and hard inclusions (refer to Table 2).

Letting the inclusion modulus $G^{\prime} \rightarrow \infty$, the present method provides the results for materials with doubly periodic rigid inclusions.

\section{Conclusions}

Using the equivalent inclusion technique integrated with the new results for doubly quasiperiodic Riemann boundary value problems, a rigorous analytical method is developed for the problem of doubly periodic cylindrical inclusions under longitudinal shear, which provides an efficient tool for the analysis of heterogeneous mate- rials (such as fiber-reinforced composites). The present method can provide benchmark results for other numerical and approximate methods, and they may be useful in the computer-aid design of new materials.

An exact solution and several illustrative examples are given. Numerical results show some expected and unexpected interesting phenomena of inclusions (fibers) interaction. In general, the influence of inclusion array pattern on stress concentration is much stronger than that on the effective modulus. In the case of multiple inclusion interaction, the use of only an inclusion density parameter (such as volume fraction) is insufficient, the parameters of inclusion distribution and geometry should be considered and extracted.

\section{Acknowledgements}

The work is supported by the Hong Kong Research Grants Council under Project HKU7011/01 E, the National Natural Science Foundation of 
China under Grant NNSFC 10272009 and the Science Foundation of Aviation of China under Grant 99G51022.

\section{References}

Accorsi, M.L., Nemat-Nasser, S., 1986. Bounds on the overall elastic and instantaneous elastoplastic moduli of periodic composites. Mechanics of Materials 5 (3), 209-220.

Adam, D.F., Doner, D.R., 1967. Longitudinal shear loading of a unidirectional composite. Journal of Composite Materials $1(1), 4$.

Bravo-Castillero, J., Guinovart-Diaz, R., Sabina, F.J, et al., 2001. Closed-form expressions for the effective coefficients of a fiber-reinforced composite with transversely isotropic constituents-II. Piezoelectric and square symmetry. Mechanics of Materials 33 (4), 237-248.

Chen, C.H., 1970. Fiber-reinforced composites under longitudinal shear loading. ASME Journal of Applied Mechanics 37, 198-201.

Chibrikova, L.I., 1956. On Riemann boundary problem for automorphic functions. Journal of Kazan University 116 (4), 59-109 (in Russian).

Chibrikova, L.I., 1970. Basic boundary problems. Lecture Notes in Kazan University.

Eshelby, J.D., 1957. The determination of the elastic field of an ellipsoidal inclusion, and related problems. Proceedings of the Royal Society of London, Series A 241, 376-396.

Furuhashi, R., Kinoshita, N., Mura, T., 1981. Periodic distribution of inclusions. International Journal of Engineering Science 19, 231-236.

Helsing, J., 1995. An integral equation method for elastostatics of periodic composites. Journal of the Mechanics and Physics of Solids 43 (6), 815-828.

Jiang, C.P., Cheung, Y.K., 1998. A fiber/matrix/composite model with a combined confocal elliptical cylinder unit cell for predicting the longitudinal shear modulus. International Journal of Solids and Structures 35 (30), 3977-3987.

Jiang, C.P., Cheung, Y.K., 2001. An exact solution for the three-phase piezoelectric cylinder model under antiplane shear and its applications to piezoelectric composites.
International Journal of Solids and Structures 38, 47774796.

Jiang, C.P., Tong, Z.H., Cheung, Y.K., 2001. A generalized self-consistent method for piezoelectric fiber reinforced composites under antiplane shear. Mechanics of Materials 33, 295-308.

Li, X., 1999. Application of doubly quasi-periodic boundary value problems in elasticity theory, Ph.D. thesis, Berlin University.

Lu, J.K., 1993. Boundary Value Problems for Analytic Functions. World Scientific, Singapore.

Muskhelishvili, N.I., 1956. Some Basic Problems of the Mathematical Theory of Elasticity. Noordhoff, Groningen (Translated from the 3rd Russian edition by Radok, J.R.M.).

Muskhelishvili, N.I., 1958. Singular Integral Equations. Noordhoff Leyden. (Translated from the Russian by Radok, J.R.M.).

Nemat-Nasser, S., Hori, M., 1999. Micromechanics: Overall Properties of Heterogeneous Materials. Elsevier Science, Amsterdam.

Nemat-Nasser, S., Taya, M., 1981. On effective moduli of an elastic body containing periodically distributed voids. Quarterly of Applied Mathematics 39, 43-59.

Nemat-Nasser, S., Taya, M., 1985. On effective moduli of an elastic body containing periodically distributed voids: comments and corrections. Quarterly of Applied Mathematics 43, 187-188.

Nunan, K.C., Keller, J.B., 1984. Effective Elasticity tensor of a periodic composite. Journal of the Mechanics and Physics of Solids 32 (4), 259-280.

Rodríguez-Ramos, R., Sabina, F.J., Guinovart-Díaz, R., BravoCastillero, J., 2001. Closed-form expressions for the effective coefficients of a fiber-reinforced composite with transversely isotropic constituents-I. Elastic and square symmetry. Mechanics of Materials 33 (4), 223-235.

Tadmor, E.B., Phillips, R., Oritz, M., 2000. Hierarchical modeling in the mechanics of materials. International Journal of Solids and Structures 37, 379-389.

Tsai, S.W., 1966. Mechanics of composite materials, part 1introduction. Air Force Materials Laboratory Contractor Report AFML-TR-66-149.

Walker, K.P., Freed, A.D., Jordan, E.H., 1991. Microstress analysis of periodic composites. Composites Engineering 1, 29-40. 